\title{
Cannabis Smoking and Periodontal Disease Among Young
}

\section{Adults}

\author{
W. Murray Thomson, PhD, Richie Poulton, PhD, Jonathan M. Broadbent, BDS, Terrie E. \\ Moffitt, PhD, Avshalom Caspi, PhD, James D. Beck, PhD, David Welch, PhD, and Robert J. \\ Hancox, MD \\ Department of Oral Sciences, Sir John Walsh Research Institute, School of Dentistry (Dr \\ Thomson and Mr Broadbent), and Department of Preventive and Social Medicine, Dunedin \\ School of Medicine (Drs Poulton, Welch, and Hancox), Dunedin, New Zealand; Social, Genetic, \\ and Developmental Psychiatry Centre, Institute of Psychiatry, King's College London, England \\ (Drs Moffitt and Caspi); Departments of Psychology and Neuroscience and Psychiatry and \\ Behavioral Science, and Institute for Genome Sciences \& Policy, Duke University, Durham, North \\ Carolina (Drs Moffitt and Caspi); and Department of Dental Ecology, School of Dentistry, \\ University of North Carolina, Chapel Hill (Dr Beck).
}

\section{Abstract}

Context-Tobacco smoking is a recognized behavioral risk factor for periodontal disease (through its systemic effects), and cannabis smoking may contribute in a similar way.

Objective-To determine whether cannabis smoking is a risk factor for periodontal disease.

Design and Setting-Prospective cohort study of the general population, with cannabis use determined at ages 18,21, 26, and 32 years and dental examinations conducted at ages 26 and 32 years. The most recent data collection (at age 32 years) was completed in June 2005.

Participants-A complete birth cohort born in 1972 and 1973 in Dunedin, New Zealand, and assessed periodically (with a $96 \%$ follow-up rate of the 1015 participants who survived to age 32 years). Complete data for this analysis were available from 903 participants (comprising $89.0 \%$ of the surviving birth cohort).

Corresponding Author: W. Murray Thomson, PhD, Department of Oral Sciences, Sir John Walsh Research Institute, School of Dentistry, PO Box 647, Dunedin, New Zealand (murray.thomson@otago.ac.nz).

Author Contributions: Dr Thomson had full access to all of the data in the study and takes responsibility for the integrity of the data and the accuracy of the data analysis.

Study concept and design: Thomson, Poulton, Moffitt, Caspi.

Acquisition of data: Thomson, Poulton, Broadbent, Moffitt, Caspi.

Analysis and interpretation of data: Thomson, Poulton, Broadbent, Beck, Welch, Hancox.

Drafting of the manuscript: Thomson, Broadbent, Welch.

Critical revision of the manuscript for important intellectual content: Thomson, Poulton, Broadbent, Moffitt, Caspi, Beck, Welch,

Hancox.

Statistical analysis: Thomson, Beck, Welch, Hancox.

Obtained funding: Thomson, Poulton, Moffitt, Caspi.

Administrative, technical, or material support: Poulton, Broadbent.

Financial Disclosures: None reported.

Additional Contributions: We thank the study members, their families, and their friends for their continuing support.

And from the standpoint of medicine as an art for the prevention and cure of disease, the man who translates the hieroglyphics of science into the plain language of healing is certainly the more useful.

-Sir William Osler (1849-1919) 
Main Outcome Measure-Periodontal disease status at age 32 years (and changes from ages 26 to 32 years) determined from periodontal combined attachment loss (CAL) measured at 3 sites per tooth.

Results-Three cannabis exposure groups were determined: no exposure (293 individuals, or $32.3 \%$ ), some exposure $(428 ; 47.4 \%)$, and high exposure $(182 ; 20.2 \%)$. At age 32 years, 265 participants $(29.3 \%)$ had 1 or more sites with $4 \mathrm{~mm}$ or greater CAL, and 111 participants $(12.3 \%)$ had 1 or more sites with $5 \mathrm{~mm}$ or greater CAL. Incident attachment loss between the ages of 26 and 32 years in the none, some, and high cannabis exposure groups was $6.5 \%, 11.2 \%$, and $23.6 \%$, respectively. After controlling for tobacco smoking (measured in pack-years), sex, irregular use of dental services, and dental plaque, the relative risk estimates for the highest cannabis exposure group were as follows: 1.6 (95\% confidence interval [CI], 1.2-2.2) for having 1 or more sites with $4 \mathrm{~mm}$ or greater CAL; 3.1 (95\% CI, 1.5-6.4) for having 1 or more sites with $5 \mathrm{~mm}$ or greater CAL; and 2.2 (95\% CI, 1.2-3.9) for having incident attachment loss (in comparison with those who had never smoked cannabis). Tobacco smoking was strongly associated with periodontal disease experience, but there was no interaction between cannabis use and tobacco smoking in predicting the condition's occurrence.

Conclusion-Cannabis smoking may be a risk factor for periodontal disease that is independent of the use of tobacco.

Periodontal disease (periodontitis) is one of the most common chronic diseases in adults; it is bacterially mediated inflammation that extends deep into the tissues, causing loss of supporting connective tissue and alveolar bone. ${ }^{1}$ Left unchecked in susceptible individuals, it can result in the loosening and eventual loss of teeth. It is second only to dental caries as a cause of tooth loss among adults in developed countries. ${ }^{2}$ Tobacco smoking is recognized as the primary behavioral risk factor for the condition. ${ }^{3,4}$ Its effect on the periodontium occurs systemically through the adverse effects of nicotine and other toxic constituents on immune function and the inflammatory response, as well as through reducing peripheral blood flow. ${ }^{5}$

Tobacco smoking has been estimated to contribute at least half of the observed variance in the condition's occurrence. ${ }^{6,7}$ Periodontal disease is understood to be a dynamic phenomenon with cyclical patterns of progression and resolution ${ }^{8}$ at any given site. Smoking is thought to tip the balance toward progression by impairing the immune response and compromising the periodontal tissue's ability to heal following a period of disease activity. ${ }^{4}$ Although a high proportion of the remaining variation can be ascribed to genetic differences, ${ }^{9}$ some can also be attributed to other environmental contributors. The deeper inhalation and prolonged contact and absorption time associated with cannabis smoking suggests that it may also be a likely candidate in the etiology of periodontal disease. Investigating such an association is challenging because of the confounding potential of concurrent tobacco smoking. ${ }^{10}$ Because of its capacity for measuring the relevant exposures without recall bias, the prospective cohort study may be the most efficacious approach for investigating the relationship between cannabis smoking and periodontal disease. We investigated the independent contributions of cannabis smoking and tobacco smoking to periodontal disease in the context of a prospective cohort study design.

\section{METHODS}

The Dunedin Multidisciplinary Health and Development Study is a longitudinal study of a cohort of children born at the Queen Mary Hospital, Dunedin, New Zealand, between April 1, 1972, and March 31, 1973.11 That institution was Dunedin's only obstetric hospital. The sample that formed the basis for the longitudinal study was 1037 children, all assessed within a month of their third birthdays, and represents $91.0 \%$ of consecutive births. The participants' families represented the full range of socioeconomic status. Periodic health and 
developmental data collections (including dental examinations) have since been undertaken. This study uses data collected from participants at ages 18, 21, 26, and 32 years. More than $90 \%$ of the cohort self-identified at age 32 years as being of European origin. Ethical approval was obtained from the Otago ethics committee, and written informed consent was obtained from each participant. The most recent data collection (at age 32 years) was completed in June 2005.

Dental examinations conducted at age 26 years included periodontal measurements made in 2 quadrants. Three sites (mesiobuccal, buccal, and distolingual) per tooth were examined, and gingival recession (the distance in millimeters from the cementoenamel junction to the gingival margin) and probing depth (the distance from the probe tip to the gingival margin) were recorded using a National Institute of Dental Research probe. Periodontal measurements were not conducted on those reporting a history of cardiac valvular anomalies or rheumatic fever (15 individuals). The combined attachment loss (CAL) for each site was computed by summing gingival recession and probing depth (third molars were not included). The dental examinations were repeated 6 years later at age 32 years. The clinical procedures were identical except that a full-mouth examination was undertaken. For changes in periodontal attachment over time (based on half-mouth data from both examinations), an incident case was an individual with 1 or more sites experiencing an increase in CAL of at least $3 \mathrm{~mm}$.

\section{Measurement of Cannabis Exposure}

At ages 18, 21, 26, and 32 years, participants were asked how many times in the previous year they had used cannabis. The exposure measure used in the current study was the mean of the usage over those 4 ages (for which Cronbach $\alpha$ was .79); participants were then assigned to 1 of 3 distributionally based cannabis exposure groups: the no exposure group, comprising those who reported 0 occasions of cannabis use; the some exposure group (a mean of $1-40$ occasions of cannabis use during the previous year); and the high exposure group, who were those with a mean of 41 or more occasions of cannabis use during the previous year (the highest 20\%). Although the usual method of smoking cannabis in New Zealand is not to mix it with tobacco, ${ }^{12}$ we cannot be absolutely certain that this was the case for all participants.

\section{Measurement of Tobacco Smoking}

The number of pack-years exposure (that is, the number of packs of cigarettes smoked per day multiplied by the number of years smoked at that rate) was computed from tobacco usage data collected for the following periods: up to age 18 years; from ages 18 to 21 years; from ages 21 to 26 years; and from ages 26 to 32 years. If data were not collected from a participant at an assessment, his or her responses at the next assessment were used and calculations made retrospectively.

\section{Other Measures}

Each participant's adult socioeconomic status (SES) was measured at age 32 years by categorizing his or her adult occupation using standard New Zealand occupation-based indices, ${ }^{13}$ which employ a 6-interval classification (where, for example, a physician scores 1 and a laborer scores 6 ). Those with a score of 5 or 6 were categorized as having low SES. To determine their usual dental service use pattern, participants were asked (at ages 26 and 32 years) whether they usually visited the dentist for a checkup or because of a problem. The latter response given at both ages identified "episodic users" of dental care. Dental plaque accumulation at age 32 years was measured using the Simplified Oral Hygiene Index, ${ }^{14}$ which scores 6 index teeth as 0 for no plaque detectable with an explorer, 1 for plaque covering not more than the cervical third of the tooth surface, 2 for plaque covering more 
than one-third but not more than two-thirds, or 3 for plaque extending over more than twothirds of the exposed tooth surface. The Simplified Oral Hygiene Index score was computed as the sum of the scores divided by the number of teeth scored.

\section{Statistical Analysis}

Bivariate analyses were conducted using SPSS version 14.0 (SPSS Inc, Chicago, Illinois), and multivariate analyses used Stata version 10.0 (StataCorp, College Station, Texas). The analysis began with univariate descriptive statistics for the cohort characteristics, periodontal attachment loss occurrence, and putative risk markers and risk factors. The bivariate analysis examined (in the following order) putative risk markers and risk factors by the cohort characteristics, the occurrence of periodontal attachment loss by the cohort characteristics, and the occurrence of periodontal attachment loss by the putative risk markers and risk factors. $\chi^{2}$ Tests were used to examine the statistical significance of differences observed with categorical dependent variables; analysis of variance was used for continuous variables. The threshold for statistical significance was $P<.05$. In the multivariate analyses, the GLM command in Stata was used with a modified Poisson approach to estimate the relative risk and confidence intervals by using robust error variances. This was used to examine the association between cannabis exposure and periodontitis prevalence (using 2 case definitions of different stringency: 1 or more sites with $4 \mathrm{~mm}$ or greater CAL, and 1 or more sites with $5 \mathrm{~mm}$ or greater $\mathrm{CAL}$ ) and incidence while controlling for tobacco smoking (pack-years), sex, SES, dental service use, and oral cleanliness. Except for cigarette smoking, those covariates were chosen because they had either been found in earlier studies to be confounders of the smoking-periodontitis relationship ${ }^{5}$ or they were associated $(P<.05)$ with the dependent variables in the bivariate analyses.

The population attributable risk from cannabis smoking between ages 18 and 32 years was calculated for the incidence of periodontal disease using 2 incident case definitions: the first used the incidence of new cases of $4 \mathrm{~mm}$ or greater CAL between ages 26 and 32 years; the second used the incidence of new cases of $5 \mathrm{~mm}$ or greater CAL between ages 26 and 32 years. For each (19.3\% and $10.4 \%$, respectively), we identified those individuals who were not cases at 26 years but were cases at age 32 years. This differs from the incident case definition used elsewhere in the article, which reflects current practice in longitudinal periodontal research. In current practice, because each individual has more than 1 site (in fact, the mean [SD] number of measured sites at age 32 years was 80 [6] with a range from 27-84), incident cases are identified as those experiencing either (or both) incident disease (that is, new attachment loss at a site that previously had had none) or progression (that is, further attachment loss at a site that had already experienced some). The computation of the population attributable risk (expressed as a percentage) required identifying and using only the new cases, and it was computed as the number exposed multiplied by the difference between incidence rates in those exposed and not exposed and then divided by the total number in the sample multiplied by their incidence rate.

\section{RESULTS}

At age 32 years, 1015 of the 1037 original participants (97.9\%) were still alive, and periodontal examination data were available for 915 . Cannabis smoking history information from at least 2 assessments between ages 18 and 32 years was available for 903 (98.7\%) of those individuals, and all subsequent analyses were limited to those 903. A comparison of the characteristics of those included and the remainder of the baseline cohort is presented in Table 1. Individuals with low SES were underrepresented among the participants included in the analyses. 
Men made up just over half of those included in the current analysis (Table 2) as did individuals with medium SES. One-third of the participants were episodic users of dentistry at both 26 and 32 years of age.

Membership of the cannabis exposure groups was as follows: there were 293 (32.3\%) in the none group ( 0 occasions of cannabis use), 428 (47.4\%) in the some group (1-40 occasions), and $182(20.2 \%)$ in the high group (41 or more occasions).

The prevalence of regular cannabis use (defined as at least weekly) more than doubled from age 18 to age 26 years and then had fallen slightly by age 32 years (Table 2). Cannabis users were more likely to be male, to occupy positions of low SES, and less likely to make dental visits. Mean plaque scores at age 32 years were significantly higher among those who used cannabis frequently at that age than among the other exposure groups (1.02 and 0.72, respectively; $P<.001)$.

Overall, half of the cohort (451, or 49.9\%) had never smoked tobacco; one-third smoked it at age 32 years $(298$, or $33.0 \%)$, and the remaining $154(17.1 \%)$ were ex-smokers. Data on cumulative exposure to tobacco smoking are presented in Table 3 by weekly use of cannabis at each age. At each age, frequent cannabis smokers were more likely to smoke tobacco. Those who were regular cannabis smokers at all of the assessment ages had the greatest tobacco exposure.

One or more periodontal sites with $4 \mathrm{~mm}$ or greater CAL were observed in 265 participants (29.3\%) at age 32 years (Table 4 ). Approximately 1 in 8 were identified as cases using the more stringent case definition of 1 or more sites with 5-mm CAL. A marked sex difference seen with the less stringent case definition was less apparent with the $5 \mathrm{~mm}$ or greater CAL criterion. There were marked SES gradients in periodontitis prevalence, with low-SES participants most affected (and those of high SES the least affected). Disease prevalence was higher among episodic dental service users than those who were not. Cannabis use was strongly associated with periodontitis prevalence, and the greatest relative differences were seen with the $5 \mathrm{~mm}$ or greater case definition, with the prevalence among the high exposure group almost 7 times that among the none group. One in 8 participants experienced incident attachment loss. There were no significant differences by sex or SES, but there were by dental service use and by cannabis use; the highest incidence was seen in the high exposure group.

When regression analysis was used to control for the confounding factors of tobacco smoking exposure, sex, SES, irregular dental service use, and the amount of plaque present (Table 5), the relative risk of having 1 or more sites with $4 \mathrm{~mm}$ or greater CAL for those who were in the highest cannabis exposure group was 1.61 (95\% confidence interval, 1.162.24) (in comparison with those who had never smoked cannabis). When the more stringent case definition ( 1 or more sites with $5 \mathrm{~mm}$ or greater CAL) was used, it was 3.13 (95\% confidence interval, 1.53-6.38). The regression analyses were repeated with only those who had never smoked tobacco (and thus without tobacco exposure in the models).

For those in the highest $20 \%$ of cannabis exposure, the relative risk estimates were 2.17 (95\% confidence interval, 1.17-4.04) for 1 or more sites with $4 \mathrm{~mm}$ or greater CAL and 4.55 (95\% confidence interval, 1.33-15.64) for 1 or more sites with $5 \mathrm{~mm}$ or greater CAL. Tobacco smoking was also strongly associated with the condition: for each increase by 1 unit in the number of pack-years, the relative risk increased between $4 \%$ and $6 \%$, depending on the dependent variable. However, there was no interaction detected between tobacco smoking and cannabis use in predicting periodontitis ( $P$ values for the interaction terms in the 3 models were $.26, .12$, and .80 , respectively). An increase by 1.0 in the plaque score (representing another one-third of the surface of the teeth covered with plaque) was 
associated with a $22 \%$ greater relative risk for having 1 or more sites with $4 \mathrm{~mm}$ or greater CAL.

As a validity check, all regression analyses were repeated using the top quartile and then the highest $10 \%$ for cannabis use (with all other variables remaining unchanged), and cannabis exposure remained a highly significant predictor in each model (data available on request). In addition, the analyses were repeated using the extent and severity of periodontitis at age 32 years. The former refers to the percentage of measured sites with $4 \mathrm{~mm}$ or greater CAL or $5 \mathrm{~mm}$ or greater CAL $(2.0 \%$ and $0.2 \%$, respectively, of a mean 80 measured sites). The latter refers to the mean CAL for sites with $2 \mathrm{~mm}$ or greater CAL. The extent and severity of periodontal disease were significantly greater among the heaviest cannabis users (Table 6).

To determine the contribution of the length of exposure to the association, we also repeated the multivariate analyses using similarly constructed cannabis exposure variables based only on ages 26 and 32 years, and the relative risks for the highest $20 \%$ of cannabis exposure were 1.49 (95\% confidence interval, 1.13-1.97) for 1 or more sites with $4 \mathrm{~mm}$ or greater CAL and 2.42 (95\% confidence interval, 1.41-4.13) for 1 or more sites with $5 \mathrm{~mm}$ or greater CAL.

Finally, regression analyses controlling for the confounding factors showed that the relative risk of developing attachment loss between ages 26 and 32 years was 2.15 for those who were in the highest cannabis exposure group (Table 5). The population attributable risk for new cases of $4 \mathrm{~mm}$ or greater CAL was $22.6 \%$ while that for the more stringent case definition of $5 \mathrm{~mm}$ or greater CAL was $36.0 \%$ (meaning that more than one-third of the new cases between ages 26 and 32 years were attributable to cannabis smoking).

\section{COMMENT}

This prospective cohort study set out to determine whether cannabis smoking was a risk factor for periodontal disease among young adults. We found that, after controlling for tobacco smoking (the most important behavioral predictor) and other important confounders, regular exposure to cannabis smoke was strongly associated with the prevalence and incidence of periodontal attachment loss by age 32 years.

Before considering the findings, it is appropriate to examine the study's limitations. First, it is possible that the prevalence of smoking at each age was underreported because of the reliance on self-reported smoking exposure data. However, a systematic review of the literature found high levels of concordance between self-report and biological measures of smoking exposure, particularly where (as in the current study) data are collected prospectively and interviewer-administered questionnaires are used in data collection. ${ }^{15}$ This has been supported by more recent studies. ${ }^{16-18}$ In any case, nondifferential underreporting of either cannabis or tobacco smoking would have resulted in more conservative estimates of the association with periodontal disease, so our findings are unlikely to be spurious.

Second, we measured periodontal attachment loss at only 3 sites per tooth (instead of 6). This will have led to some underestimation of both prevalence and incidence, but its magnitude is unknown. However, a recent study of the effect of partial recording protocols on estimates of periodontal disease prevalence found that the particular 3-site combination used in the current study was associated with the least misclassification when compared with estimates from the use of all 6 sites per tooth. ${ }^{19} \mathrm{We}$ were unable to determine whether any participant had had periodontal surgery by age 32 years (which could possibly have eliminated periodontal pocketing). 
Among the study's strengths are the very high follow-up rates in the longitudinal study, the prospective determination of smoking exposure (and the length of time over which those exposure data were collected), and the use of data on periodontal disease incidence as well as prevalence. Moreover, the typical pattern of cannabis use in New Zealand does not involve mixing it with tobacco, and this is likely to have resulted in a "cleaner" cannabis exposure variable. The generalizability of the findings to the source population has been established, and we addressed in an earlier report ${ }^{20}$ the issue of the degree to which they can be generalized to similar populations in both New Zealand and the United States; essentially, the baseline (age 26 years) peridontal estimates from our study were broadly comparable with those reported from other representative samples in New Zealand and the United States.

The study's demonstration of a strong association between cannabis use and periodontitis experience by age 32 years indicates that long-term smoking of cannabis is detrimental to the periodontal tissues and that public health measures to reduce the prevalence of cannabis smoking may have periodontal benefits for the population. To our knowledge, no previous studies have examined this relationship, so there are no data with which to compare the findings. Determining whether the association exists in other populations should be a priority for periodontal epidemiological research. The nature of the biological mechanism for the observed association is currently unclear. The periodontal effects of tobacco smoke are thought to occur via the systemic effects of nicotine and other toxic constituents on immune function and the inflammatory response within the periodontal tissues. Cannabis contains more than 400 compounds, including more than 60 cannabinoids; the noncannabinoid constituents are similar to tobacco (except for nicotine), and those have been reported to carry systemic health risks and have histopathological effects that are similar to those of tobacco smoke. ${ }^{21,22}$

Although definitively establishing the periodontal effects of exposure to cannabis smoke should await confirmation in other populations and settings, health promoters and dental and medical practitioners should take steps to raise awareness of the strong probability that regular cannabis users may be doing damage to the tissues that support their teeth.

\section{Acknowledgments}

Funding/Support: This work was supported by grant R01 DE-015260 from the National Institute of Dental and Craniofacial Research; grants MH45070, MH49414, and MH077874 from the National Institute of Mental Health; grant G0100527 from the Medical Research Council of the United Kingdom; and a program grant from the Health Research Council of New Zealand. The Dunedin Multidisciplinary Health and Development Research Unit is supported by the Health Research Council of New Zealand.

Role of the Sponsors: The sponsors had no role in the design and conduct of the study; the collection, management, analysis, and interpretation of the data; or the preparation, review, or approval of the manuscript.

\section{References}

1. Pihlstrom BL, Michalowicz BS, Johnson NW. Periodontal diseases. Lancet 2005;366(9499):18091820. [PubMed: 16298220]

2. Phipps KR, Stevens VJ. Relative contribution of caries and periodontal disease in adult tooth loss for an HMO dental population. J Public Health Dent 1995;55(4):250-252. [PubMed: 8551465]

3. Gelskey SC. Cigarette smoking and periodontitis: methodology to assess the strength of evidence in support of a causal association. Community Dent Oral Epidemiol 1999;27(1):16-24. [PubMed: 10086922]

4. Johnson GK, Hill M. Cigarette smoking and the periodontal patient. J Periodontol 2004;75(2):196209. [PubMed: 15068107] 
5. Kinane DF, Chestnutt IG. Smoking and periodontal disease. Crit Rev Oral Biol Med 2000;11(3): 356-365. [PubMed: 11021635]

6. Tomar SL, Asma S. Smoking-attributable periodontitis in the United States: findings from NHANES III, National Health and Nutrition Examination Survey. J Periodontol 2000;71(5):743751. [PubMed: 10872955]

7. Hujoel PP, del Aguila MA, DeRouen TA, Bergstrom J. A hidden periodontitis epidemic during the 20th century? Community Dent Oral Epidemiol 2003;31(1):1-6. [PubMed: 12542426]

8. Gilthorpe MS, Zamzuri AT, Griffiths GS, Maddick IH, Eaton KA, Johnson NW. Unification of the "burst" and "linear" theories of periodontal disease progression: a multilevel manifestation of the same phenomenon. J Dent Res 2003;82(3):200-205. [PubMed: 12598549]

9. Yoshie H, Kobayashi T, Tai H, Galicia JC. The role of genetic polymorphisms in periodontitis. Periodontol 2000 2007;43:102-132. [PubMed: 17214838]

10. Johnson N. Tobacco use and oral cancer: a global perspective. J Dent Educ 2001;65(4):328-339. [PubMed: 11336118]

11. Silva, PA.; Stanton, WR. From Child to Adult: the Dunedin Multidisciplinary Health and Development Study. Auckland, New Zealand: Oxford University Press; 1996.

12. Aldington S, Williams M, Nowitz M, et al. Effects of cannabis on pulmonary structure, function and symptoms. Thorax 2007;62(12):1058-1063. [PubMed: 17666437]

13. Irving JC, Elley WB. A socio-economic index for the female labour force in New Zealand. N Z J Educ Stud 1977;12:154-163.

14. Greene JC, Vermillion JR. The Simplified Oral Hygiene Index. J Am Dent Assoc 1964;68:7-13. [PubMed: 14076341]

15. Patrick DL, Cheadle A, Thompson DC, Diehr P, Koepsell T, Kinne S. The validity of self-reported smoking: a review and meta-analysis. Am J Public Health 1994;84(7):1086-1093. [PubMed: 8017530]

16. Mak YW, Loke AY, Lam TH, Abdullah AS. Validity of self-reports and reliability of spousal proxy reports on the smoking behavior of Chinese parents with young children. Addict Behav 2005;30(4):841-845. [PubMed: 15833586]

17. Fendrich M, Mackesy-Amiti ME, Johnson TP, Hubbell A, Wislar JS. Tobacco-reporting validity in an epidemiological drug-use survey. Addict Behav 2005;30(1):175-181. [PubMed: 15561458]

18. Dolcini MM, Adler NE, Lee P, Bauman KE. An assessment of the validity of adolescent selfreported smoking using three biological indicators. Nicotine Tob Res 2003;5(4):473-483. [PubMed: 12959785]

19. Susin C, Kingman A, Albandar JM. Effect of partial recording protocols on estimates of prevalence of periodontal disease. J Periodontol 2005;76(2):262-267. [PubMed: 15974851]

20. Thomson WM, Broadbent JM, Poulton R, Beck JD. Changes in periodontal disease experience from age 26 to 32 in a birth cohort. J Periodontol 2006;77(6):947-954. [PubMed: 16734567]

21. Ashton CH. Pharmacology and effects of cannabis: a brief review. Br J Psychiatry 2001;178:101106. [PubMed: 11157422]

22. Taylor DR, Hall W. Respiratory health effects of cannabis: position statement of the Thoracic Society of Australia and New Zealand. Intern Med J 2003;33(7):310-313. [PubMed: 12823677] 
Table 1

Comparison of Characteristics at Age 18 Years for Those Included and Not Included

\begin{tabular}{lccc}
\hline \multirow{2}{*}{ Characteristic at Age 18 Years } & Included & Not Included & \multirow{2}{*}{$\boldsymbol{P}$ Value } \\
\cline { 2 - 3 } Total & $903(87.1)$ & $134(12.9)^{a}$ & \\
\hline Men & $461(51.1)$ & $74(55.2)$ & .37 \\
\hline Low socioeconomic status $b$ & $175(19.5)$ & $40(30.1)$ & .005 \\
\hline Smoked cannabis at least weekly & $75(8.3)$ & $6(4.5)$ & .12 \\
\hline Tobacco use, mean (SD), pack-years & $0.65(1.13)$ & $0.95(1.42)$ & .06 \\
\hline
\end{tabular}

${ }^{a}$ Twenty-two participants had died by the assessment at age 32 years; of the remaining 112, 57 were assessed at age 32 years but did not provide dental or periodontal data, and 12 more provided dental data but not cannabis data.

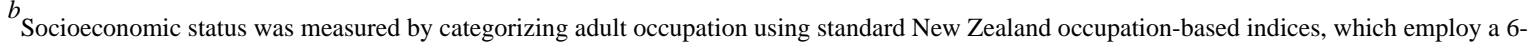
interval classification; those with a score of 5 or 6 were categorized as having low socioeconomic status. 


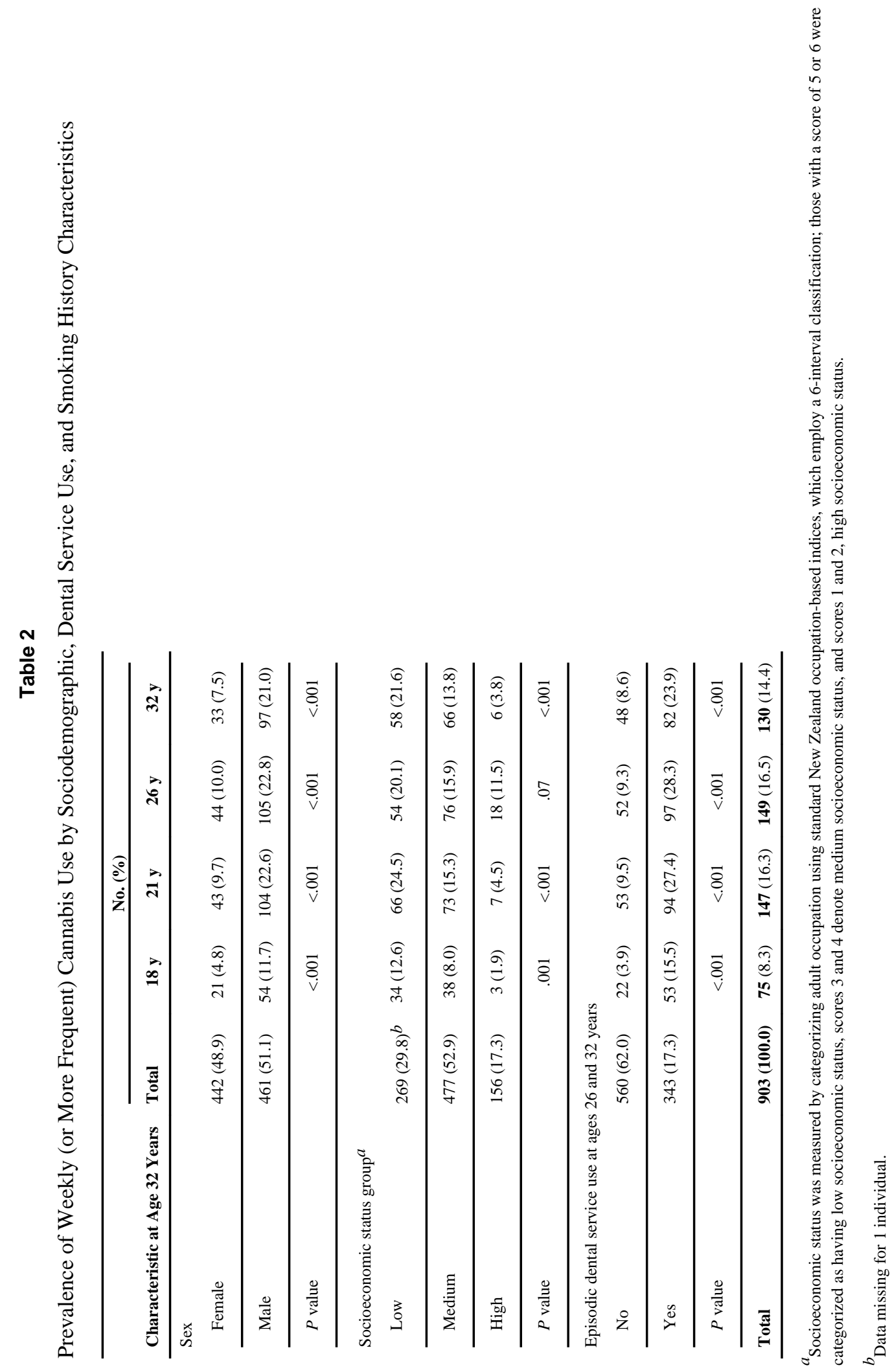


Table 3

Tobacco Smoking by Weekly Cannabis Use at Ages 18 Through 32 Years

\begin{tabular}{|c|c|c|}
\hline Cannabis Used at Least Weekly & $\begin{array}{c}\text { Cumulative Tobacco Smoking to That Age, } \\
\text { Mean (SD),Pack-Years }\end{array}$ & $\begin{array}{c}\text { Median Tobacco Smoking to That Age, Pack-Years } \\
\text { (Interquartile Range) }\end{array}$ \\
\hline \multicolumn{3}{|l|}{ Age 18 years } \\
\hline No & $0.51(0.96)^{a}$ & $0.00(0.00-0.68)$ \\
\hline Yes & $2.21(1.57)$ & $2.25(1.00-3.00)$ \\
\hline \multicolumn{3}{|l|}{ Age 21 years } \\
\hline No & $1.17(1.91)^{a}$ & $0.00(0.00-1.90)$ \\
\hline Yes & $3.48(2.68)$ & $3.05(1.20-5.50)$ \\
\hline \multicolumn{3}{|l|}{ Age 26 years } \\
\hline No & $2.43(3.58)^{a}$ & $0.00(0.00-4.50)$ \\
\hline Yes & $6.34(4.68)$ & $6.75(1.60-10.00)$ \\
\hline \multicolumn{3}{|l|}{ Age 32 years } \\
\hline No & $3.75(5.60)^{a}$ & $0.00(0.00-6.85)$ \\
\hline Yes & $10.90(7.37)$ & $12.43(4.83-16.06)$ \\
\hline \multicolumn{3}{|l|}{ At any of ages $18,21,26$, or 32 years } \\
\hline No & $3.06(5.04)^{a}$ & $0.00(0.00-4.93)$ \\
\hline Yes & $9.48(7.31)$ & $9.43(2.84-15.18)$ \\
\hline \multicolumn{3}{|l|}{ At each of ages $18,21,26$, or 32 years } \\
\hline No & $4.32(5.99)^{a}$ & $0.00(0.00-7.95)$ \\
\hline Yes & $15.32(6.37)$ & $15.98(13.46-19.81)$ \\
\hline
\end{tabular}

$a_{P<.001}$ 
Table 4

Periodontitis Prevalence at Age 32 Years and Incidence From Ages 26 to 32 Years by Sociodemographic Characteristics, Smoking History, and Exposure to Cannabis

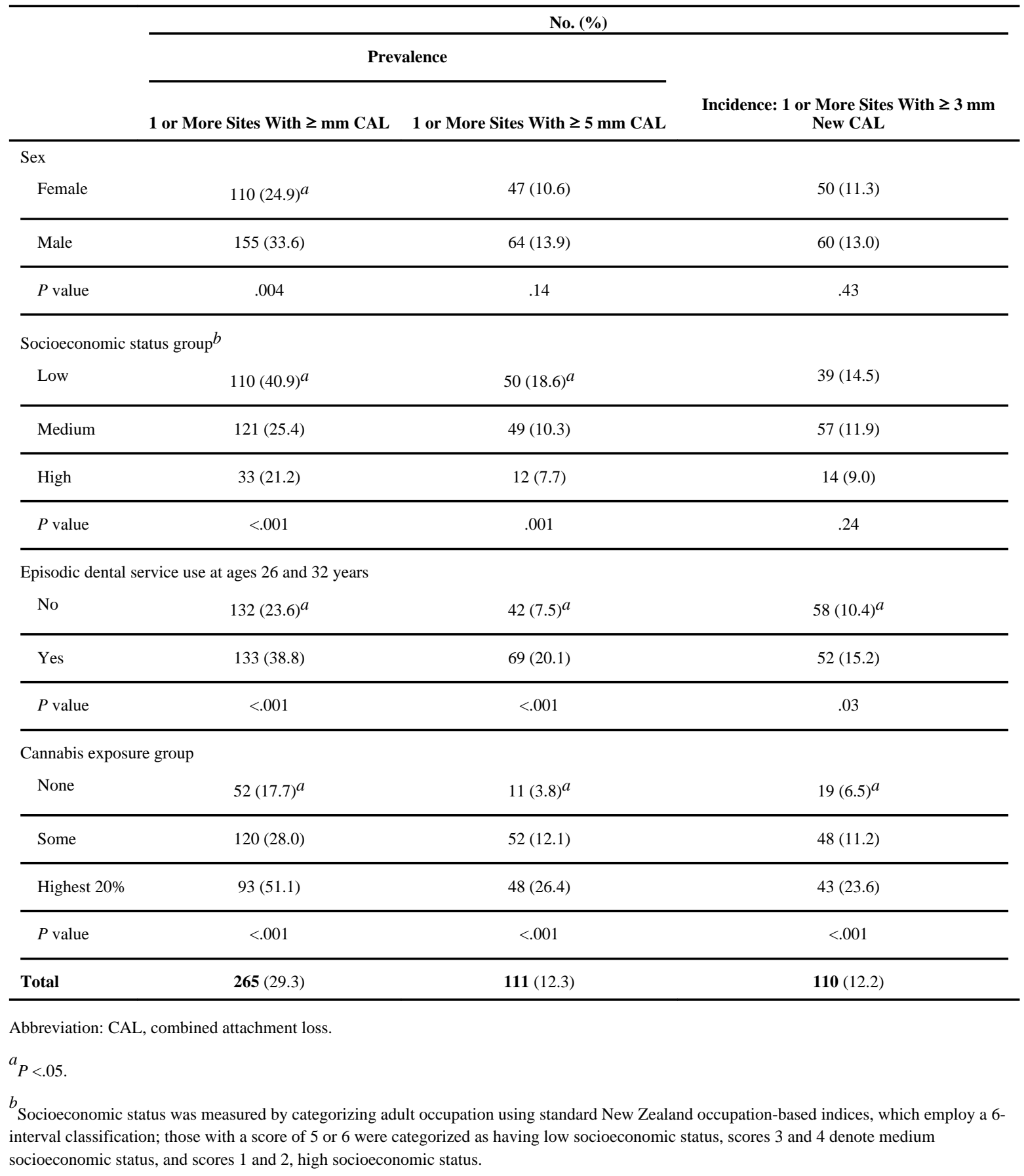


Table 5

Outcome of Multivariate Analyses of Periodontitis Prevalence and Incidence

\begin{tabular}{|c|c|c|c|}
\hline & \multicolumn{3}{|c|}{ Relative Risk (95\% Confidence Interval) } \\
\hline & \multicolumn{2}{|c|}{ Prevalence at Age 32 Years } & \multirow{2}{*}{$\begin{array}{c}\text { Incidence Between Ages } 26 \text { and } \\
32 \text { Years: } \geq 1 \text { Sites With } \geq 3 \\
\text { mm New CAL }\end{array}$} \\
\hline & $\underset{\text { CAL }}{\geq 1 \text { Sites With }} \geq 4 \mathrm{~mm}$ & $\underset{\text { CAL }}{\geq 1 \text { Sites With }} \geq 5 \mathrm{~mm}$ & \\
\hline Highest $20 \%$ of cannabis exposure $a$ & $1.61(1.16-2.24)$ & $3.13(1.53-6.38)$ & $2.15(1.20-3.85)$ \\
\hline Some cannabis exposure & $1.34(1.01-1.80)$ & $2.50(1.32-4.72)$ & $1.47(0.87-2.47)$ \\
\hline Pack-years of tobacco use, per pack year & $1.04(1.03-1.06)$ & $1.06(1.03-1.09)$ & $1.06(1.03-1.09)$ \\
\hline Female & $0.89(0.72-1.09)$ & $1.00(0.69-1.43)$ & $1.03(0.71-1.50)$ \\
\hline Low socioeconomic status $b$ & $1.23(0.87-1.74)$ & $1.31(0.70-2.44)$ & $1.00(0.54-1.84)$ \\
\hline Medium socioeconomic status & $0.98(0.70-1.36)$ & $1.02(0.56-1.84)$ & $1.07(0.61-1.87)$ \\
\hline $\begin{array}{l}\text { Episodic dental service use at ages } 26 \text { and } 32 \\
\text { years }\end{array}$ & $1.05(0.84-1.31)$ & $1.51(1.00-2.28)$ & $0.90(0.60-1.34)$ \\
\hline Plaque score at age 32 years, per unit ${ }^{c}$ & $1.22(1.01-1.46)$ & $0.99(0.69-1.40)$ & $1.00(0.69-1.45)$ \\
\hline
\end{tabular}

Abbreviation: CAL, combined attachment loss.

${ }^{a}$ Reference categories were as follows: cannabis exposure variables: none group; female: male; low or medium socioeconomic status at age 32 years: high socioeconomic status; episodic dental service use at ages 26 and 32 years: routine dental service use.

${ }^{b}$ Socioeconomic status was measured by categorizing adult occupation using standard New Zealand occupation-based indices, which employ a 6interval classification; those with a score of 5 or 6 were categorized as having low socioeconomic status, scores 3 and 4 denote medium socioeconomic status, and scores 1 and 2 , high socioeconomic status.

${ }^{c}$ This is a continuous variable representing the extent of plaque on 6 index teeth (range, 0 to 3). 
Table 6

Extent and Severity of Combined Attachment Loss by Cannabis Exposure Group

\begin{tabular}{cllll}
\hline & \multicolumn{2}{c}{ Cannabis Exposure Group, \% } & \\
\cline { 2 - 3 } & None & Some & Highest 20\% & All Combined, \% \\
\hline $\begin{array}{c}\text { Extent of 4 mm or greater CAL } \\
\text { Mean (SD) }\end{array}$ & $0.9(4.2)$ & $1.7(4.5)$ & $4.7(10.0)^{a}$ & $2.0(6.1)$ \\
\hline Median (interquartile range) & $0.0(0.0-0.0)$ & $0.0(0.0-1.2)$ & $1.2(0.0-4.8)$ & $0.0(0.0-1.2)$ \\
\hline Extent of 5 mm or greater CAL & & & & \\
Mean (SD) & $0.2(1.9)$ & $0.4(1.4)$ & $1.5(5.1)^{a}$ & $0.6(2.8)$ \\
\hline Median (interquartile range) & $0.0(0.0-0.0)$ & $0.0(0.0-0.0)$ & $0.0(0.0-1.2)$ & $0.0(0.0-0.0)$ \\
\hline Severity of CAL & & & & \\
\hline Mean (SD) & $3.1(0.2)$ & $3.1(0.2)$ & $3.3(0.4)^{a}$ & $3.1(0.3)$ \\
\hline \begin{tabular}{l} 
Median (interquartile range) \\
\hline
\end{tabular} & $3.0(3.0-3.0)$ & $3.0(3.0-3.2)$ & $3.2(3.0-3.4)$ & $3.0(3.0-3.2)$ \\
\hline
\end{tabular}

Abbreviation: CAL, combined attachment loss.

${ }^{a_{P}<.001 ; \text { Kruskal-Wallis test. }}$ 